\title{
Atrial pacing at multiple sites in the Wolff-Parkinson-White syndrome ${ }^{1}$
}

\author{
P. DENES, C. R. WYNDHAM, F. AMAT-Y-LEON, D. WU, R. C. DHINGRA, \\ R. H. MILLER, AND K. M. ROSEN \\ From the Section of Cardiology, Department of Medicine, Abraham Lincoln School of Medicine, University \\ of Illinois College of Medicine, and the West Side Veterans Administration Hospital, Chicago, \\ Illinois, U.S.A.
}

Atrial pacing at multiple sites was used in an attempt to predict the site of pre-excitation in 5 patients with Wolff-Parkinson-White syndrome with 5 different anomalous pathway locations (right anterior, right posterior, septal, left posterior, and left lateral). At least 3 atrial pacing sites were tested in each patient. Pacing sites tested included high right atrium, low lateral right atrium, low septal right atrium, proximal coronary sinus, and distal coronary sinus. Atrial stimulation sites with shortest and longest stimulus-delta intervals could be identified in each patient, the shortest stimulus-delta interval in each case ranging from 60 to $80 \mathrm{~ms}$. The difference between the shortest and longest stimulus-delta interval in each case ranged from 60 to $110 \mathrm{~ms}$. It was suggested that the site with the shortest stimulus-delta interval corresponded to a site close to the atrial insertion of the anomalous pathway. This hypothesis was confirmed in all cases (3 with epicardial mapping and 2 with retrograde atrial activation data). In conclusion, atrial pacing at multiple sites is helpful in predicting the site of anterogradely conducting anomalous pathways, and appears particularly useful for differentiation of right posterior, left posterior, and septal pre-excitation.

Cure of Wolff-Parkinson-White syndrome is now possible using epicardial mapping for delineating the site of pre-excitation, and surgical transection of anomalous AV connections (Sealy and Wallace, 1974; Gallagher et al., 1975). Anomalous pathway location plays a role in determining the success of operation (Sealy and Wallace, 1974; Gallagher et al., 1975; Tonkin et al., 1975). Right anterior, lateral, and posterior as well as left lateral anomalous AV connections appear readily accessible to surgical cure. Left posterior and septal anomalous pathways are less accessible to the surgeon. Since, therefore, the surgical cure of pre-excitation appears to be partly related to anomalous pathway location, the accurate preoperative localisation of these pathways has assumed considerable importance.

The means of predicting anomalous pathway locations include surface electrocardiography and vectorcardiography, as well as electrophysiological evaluation in the catheterisation laboratory (Wellens

\footnotetext{
'Supported in part by a USPHS Training Grant, and Basic Institutional Support of the West Side Veterans Administration Hospital, Chicago, Illinois.

Received for publication 31 August 1976
}

et al., 1971; Touboul et al., 1972; Coumel and Attuel, 1974; Denes et al., 1974; Spurrell et al., 1974; Svenson et al., 1974, 1975; Wellens and Durrer, 1974, 1975; Wyndham et al., 1974; Zipes et al., 1974; Gallagher et al., 1975; Neuss et al., 1975; Denes et al., 1976a, b). Electrophysiological techniques include lateralisation of the anomalous pathway by noting the effects of functional bundlebranch block on the cycle length of circus movement tachycardia or on retrograde anomalous pathway conduction time (Coumel and Attuel, 1974; Spurrell et al., 1974; Zipes et al., 1974; Neuss et al., 1975; Wellens and Durrer, 1975); intracardiac catheter electrode mapping of retrograde atrial activation during circus movement tachycardia and/or with ventricular pacing (Svenson et al., 1974, 1975; Wellens and Durrer, 1974, 1975; Zipes et al., 1974; Gallagher et al., 1975; Neuss et al., 1975; Denes et al., 1976a, b), and evaluation of atrial pacing at multiple sites with observation of QRS morphology and stimulus-delta intervals (Wellens et al., 1971; Touboul et al., 1972; Denes et al., 1974; Gallagher et al., 1975; Svenson et al., 1975; Denes et al., 1976a). 
Although all these techniques appear to be of value in localisation of anomalous pathways, major emphasis has been placed upon the use of retrograde atrial activation sequences in the prediction of anomalous pathway location (Zipes et al., 1974; Wellens and Durrer, 1974, 1975; Gallagher et al., 1975; Neuss et al., 1975; Svenson et al., 1975; Denes et al., 1976a). The use of atrial pacing at multiple sites has received less attention. In the present study, we describe the results of atrial pacing studies at multiple sites in 5 patients with anterogradely conducting anomalous pathways in different locations. Our results confirm the value of multiple atrial pacing sites in anomalous pathway localisation. In addition, criteria are developed for using the information obtained with this technique in predicting the site of pre-excitation.

\section{Methods}

Five patients with pre-excitation were selected for presentation in this report. All patients had typical surface electrocardiographic findings of WolffParkinson-White syndrome including a short PR interval (less than $0.12 \mathrm{~s}$ ), wide QRS complexes $(0.12 \mathrm{~s}$ or greater), delta waves, and documented supraventricular tachyarrhythmias. These 5 patients were selected because: (1) technically adequate multiple atrial pacing site data were obtained for analysis, and (2) the anomalous pathway location was reasonably well documented, so that the predictive value of multiple atrial pacing sites could be examined. (3) The patients represented 5 different anomalous pathway locations.

Electrophysiological studies were performed in the supine, non-sedated state. Informed written consent was obtained from all patients before the study. All cardioactive drugs were discontinued 48 hours before the study. Catheter techniques for recording and stimulation were similar to those previously described (Wyndham et al., 1974; Denes et al., 1976a, b). Electrocardiographic leads I, II, III, and V1, atrial and His bundle electrograms were recorded using a paper speed of $100 \mathrm{~mm} / \mathrm{s}$. Intervals were measured by averaging 10 consecutive beats. Tripolar and quadripolar catheters (with interelectrode distances of $1 \mathrm{~cm}$ ) were placed under fluoroscopic control.

The following atrial pacing sites were used: (1) high right atrium, at the superior vena cava-right atrial junction, (2) low lateral right atrium, at the inferior vena cava-right atrial junction, (3) low septal right atrium (using the His bundle recording catheter) at the most distal site on pull-back from the ventricle, where stable atrial capture with an atrial depolarisation preceding the QRS complex could be obtained, (4) right atrial appendage, (5) proximal coronary sinus (just inside the os of the coronary sinus), (6) distal coronary sinus (at the most distal site in the coronary sinus where atrial capture could be obtained, usually close to the left heart border).

The atria were stimulated from the tested sites at rates slightly faster than sinus and then in 10 beats/ minute increments until total pre-excitation was achieved. The stimulus-delta remained constant at increasing pacing rates. For purposes of comparison results in each case were compared at an identical cycle length between 460 and $600 \mathrm{~ms}$. Epicardial mapping techniques were similar to those previously described by our laboratory (Wyndham et al., 1974; Denes et al., 1976a, b).

\section{DEFINITIONS}

Pre-excitation was classified electrocardiographically during maximal pre-excitation. The electrocardiographic classification of pre-excitation was based on the work of Boineau et al. (1973).

Anterior right ventricular pre-excitation (type B) was defined by the presence of a QS or rS in V1 and an $R$ in I and III. Posterior right ventricular preexcitation (type $\mathrm{AB}$ ) was defined by the presence of a QS or rS in V1, an R in I, and a QS in III. Posterior left ventricular pre-excitation (type A) was defined by the presence of an R or RS in V1, an $R$ in $I$, and a $Q S$ or $r S$ in III. Lateral left ventricular pre-excitation (type $\mathrm{C}$ ) was defined by the presence of an R or RS in V1, a QS or rS in I, and an R in III.

Stimulus-delta intervals were measured from the onset of the stimulus artefact to the earliest onset of the delta wave on simultaneous surface electrocardiographic leads. This interval represented the atrial and anomalous pathway conduction times from the site of atrial stimulation.

Total or maximal pre-excitation referred to a QRS complex resulting from ventricular activation exclusively through the anomalous atrioventricular connection.

\section{Results}

\section{CASE 1}

The patient was a 25 -year-old woman with symptomatic recurrent paroxysmal supraventricular tachycardia and recurrent paroxysmal atrial fibrillation with rapid ventricular response. Electrocardiograms revealed type B pre-excitation (Fig. 1). Basic conduction intervals were recorded during sinus rhythm at a cycle length of $610 \mathrm{~ms}$ and revealed a PA interval of $30 \mathrm{~ms}$. AH and HV intervals could not be measured since the His bundle electrograms were buried within the ventricular electrogram. The $P$ delta interval was of $75 \mathrm{~ms}$. 

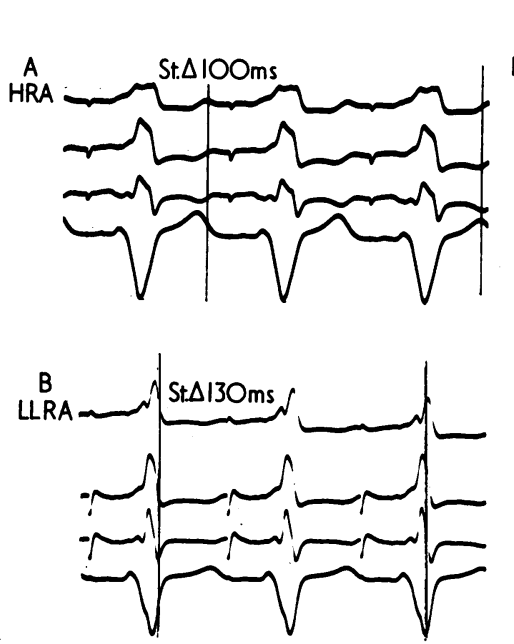
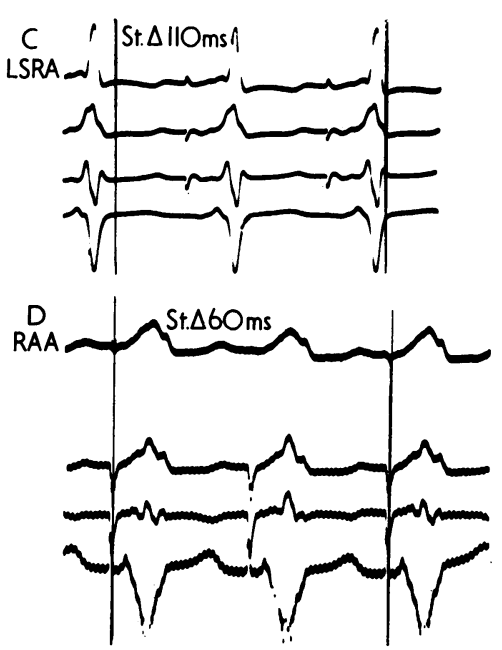

Fig. 1 The effect of atrial pacing at multiple sites on stimulus-delta intervals in case 1 with type $B$ pre-excitation. Shown in each panel from top to bottom are surface electrocardiographic leads I, II, III, and V1. HRA represents high right atrial pacing; LLRA low lateral right atrial pacing; LSRA low septal right atrial pacing; RAA right atrial appendage pacing.

Cycle length was $500 \mathrm{~ms}$. Stimulus-delta intervals are listed and expressed in $\mathrm{ms}$. Time lines are at 1 second and paper speed is $100 \mathrm{~mm} / \mathrm{s}$ in this and subsequent illustrations. Note that the shortest stimulusdelta interval $(60 \mathrm{~ms})$ is obtained with right atrial appendage pacing (panel D).
Four atrial pacing sites were studied at a cycle length of $500 \mathrm{~ms}$. The stimulus-delta intervals and QRS durations from these sites were as follows:

(1) high right atrium, with a stimulus-delta of $100 \mathrm{~ms}$ and QRS duration of $180 \mathrm{~ms}$ (Fig. 1A);

(2) low lateral right atrium, with a stimulus-delta of $130 \mathrm{~ms}$ and a QRS duration of $150 \mathrm{~ms}$ (Fig. 1B); (3) low septal right atrium with a stimulus-delta of $110 \mathrm{~ms}$ and QRS duration of $130 \mathrm{~ms}$ (Fig. 1C); (4) right atrial appendage, with a stimulus-delta of $60 \mathrm{~ms}$ and QRS duration of $180 \mathrm{~ms}$ (Fig. 1D).

Curative surgery was attempted because of recurrent tachyarrhythmias. Epicardial mapping revealed earliest ventricular activation on the anterior right ventricle adjacent to the right atrial appendage (Fig. 2). The epicardial ventricular electrograms preceded the onset of the delta wave by $38 \mathrm{~ms}$. After epicardial mapping, the right atrium was entered during cardiopulmonary bypass. A transmural atrial incision was made parallel to the $\mathrm{AV}$ ring and adjacent to the area of earliest ventricular activation. All subsequent electrograms have revealed normal PR intervals without pre-excitation. Arrhythmias have not recurred (6-month followup).

\section{Comment}

Electrocardiograms before surgery suggested a right anterior anomalous pathway. Atrial pacing studies at multiple sites provided strong evidence of an anterior right $\mathrm{AV}$ connection with demonstration of the shortest stimulus-delta interval $(60 \mathrm{~ms})$ and maximum pre-excitation from the right atrial appendage pacing site. The predicted site of preexcitation was confirmed at cardiac surgery.

\section{CASE 2}

This was a 15-year-old youth with recurrent paroxysmal supreventricular tachycardia. Electrocardiograms during total pre-excitation revealed type AB pre-excitation. This case has been previously reported because of bilateral anomalous pathways (a manifest right posterior anterograde pathway, and a left lateral concealed retrograde pathway), and will, therefore, be described only briefly here (Denes et al., 1976a).

Three atrial pacing sites were studied at a cycle length of $600 \mathrm{~ms}$ (Fig. 2 of Denes et al. (1976a)). The stimulus-delta intervals and QRS duration from these sites were as follows: (1) high right atrium, with a stimulus-delta of $140 \mathrm{~ms}$ and QRS duration of $120 \mathrm{~ms}$; (2) low lateral right atrium, with a stimulus-delta of $80 \mathrm{~ms}$ and QRS duration of $180 \mathrm{~ms}$; (3) proximal coronary sinus, with a stimulus-delta of $115 \mathrm{~ms}$ and QRS duration of $170 \mathrm{~ms}$. Epicardial mapping revealed early activation of the posterior right ventricle contiguous to the intraventricular groove, with early activation coinciding with the onset of the delta wave (Fig. 6 of Denes et al. (1976a)). A transmural right atrial incision parallel to the AV ring adjacent to the site of earliest ventricular activation resulted in cure of anterograde pre-excitation. 


\section{Comment}

In this patient, the electrocardiogram during total pre-excitation suggested a posterior right ventricular connection. The atrial pacing site study showed the shortest stimulus-delta and widest QRS complex from the low lateral right atrium suggesting a lateral right atrial connection. The posterior right atrium was not tested. Epicardial mapping studies and surgery localised the anterogradely conducting anomalous connection to the right posterior ventricle.

\section{CASE 3}

This was a 37-year-old man with recurrent paroxysmal supraventricular tachycardia. Electrocardiograms during total pre-excitation indicated type AB pre-excitation (Fig. 3).

Basic conduction intervals were recorded during sinus rhythm at a cycle length of $870 \mathrm{~ms}$ and were as follows: P delta of $100 \mathrm{~ms}$ and PA of $30 \mathrm{~ms}$. AH and $H V$ intervals could not be measured since the $\mathrm{H}$ potential was buried within the ventricular electrogram.

Four atrial pacing sites were selected for pacing at a cycle length of $460 \mathrm{~ms}$. The stimulus-delta intervals and QRS duration from these sites were as follows: (1) High right atrium, with a stimulusdelta of $110 \mathrm{~ms}$ and QRS duration of $170 \mathrm{~ms}$ (Fig. 3A). (2) Low lateral right atrium, with a stimulusdelta of $110 \mathrm{~ms}$ and QRS duration of $170 \mathrm{~ms}$ (Fig. 3B). (3) Low septal right atrium, with a stimulusdelta of $70 \mathrm{~ms}$ and QRS duration of $170 \mathrm{~ms}$ (Fig. 3C). (4) Distal coronary sinus, with a stimulusdelta of $140 \mathrm{~ms}$ and QRS duration of $170 \mathrm{~ms}$ (Fig. 3D).

Retrograde atrial activation sequences were determined during right ventricular pacing. Low right atrial septal electrograms preceded high right atrial, lateral right atrial, and right atrial appendage electrograms by 30,40 , and $50 \mathrm{~ms}$, respectively. The retrograde conduction time measured from the onset of the QRS to the low septal right atrial electrogram was $90 \mathrm{~ms}$ and was constant at all ventricular paced cycle lengths.

\section{Comment}

The electrocardiogram in this patient was similar to that of case 2 and suggested a posterior right ventricular connection. However, atrial pacing site studies strongly suggested a septal anomalous pathway, because of the very short stimulus-delta with low right atrial septal pacing. Though this anomalous pathway location was not confirmed with epicardial mapping and surgery, the retrograde activation data provided strong confirmatory evidence for this predicted site of pre-excitation
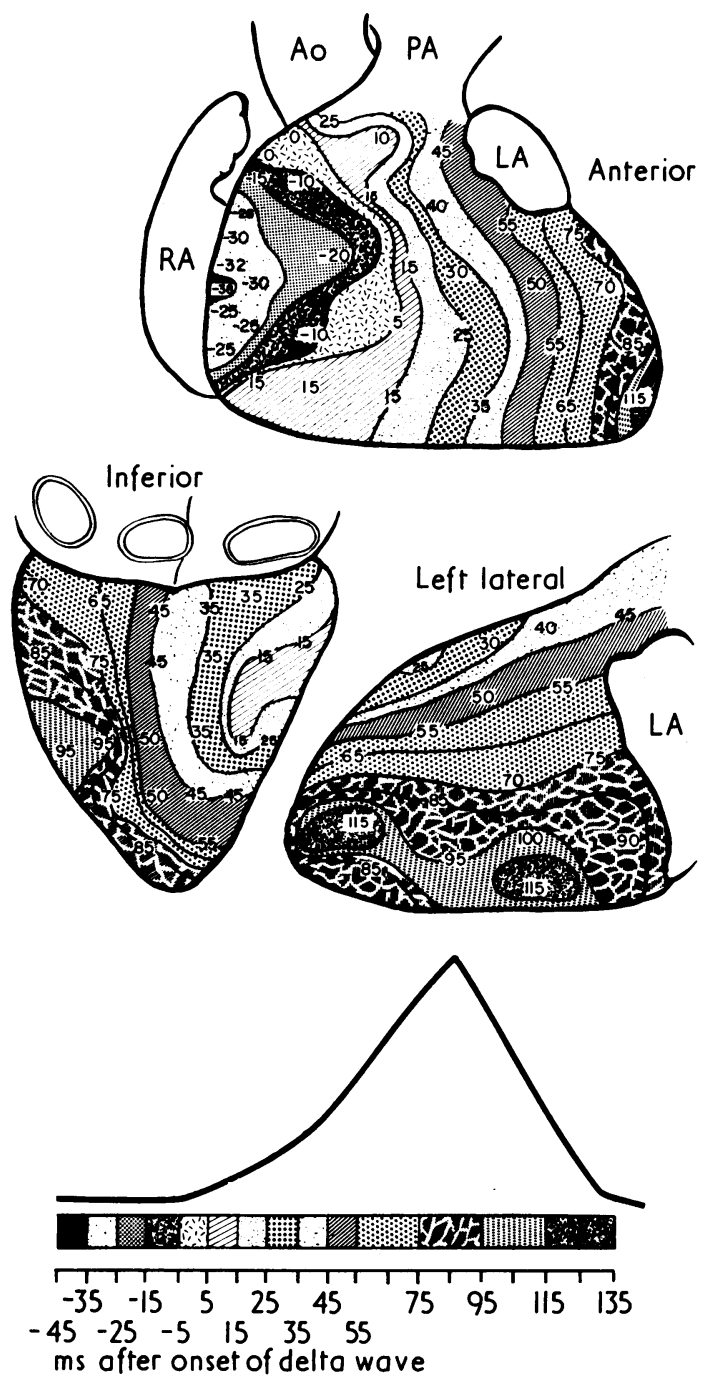

Fig. 2 Diagram of epicardial activation during preexcitation in case 1. Isochronous lines are drawn at 10 to 20 ms intervals with reference to the onset of delta wave. Inferior (upper), anterior (lower left), and left lateral (lower right) views of the heart are shown. The earliest area of epicardial activity is on the posterolateral left ventricle.

(Gallagher et al., 1975). These electrophysiological findings led to a decision not to attempt surgical cure of the pre-excitation.

CASE 4

This was an 18-year-old youth with paroxysmal atrial fibrillation. Electrocardiograms revealed type A pre-excitation (Fig. 4). Basic conduction intervals at a cycle length of $765 \mathrm{~ms}$ revealed a P delta of 120 

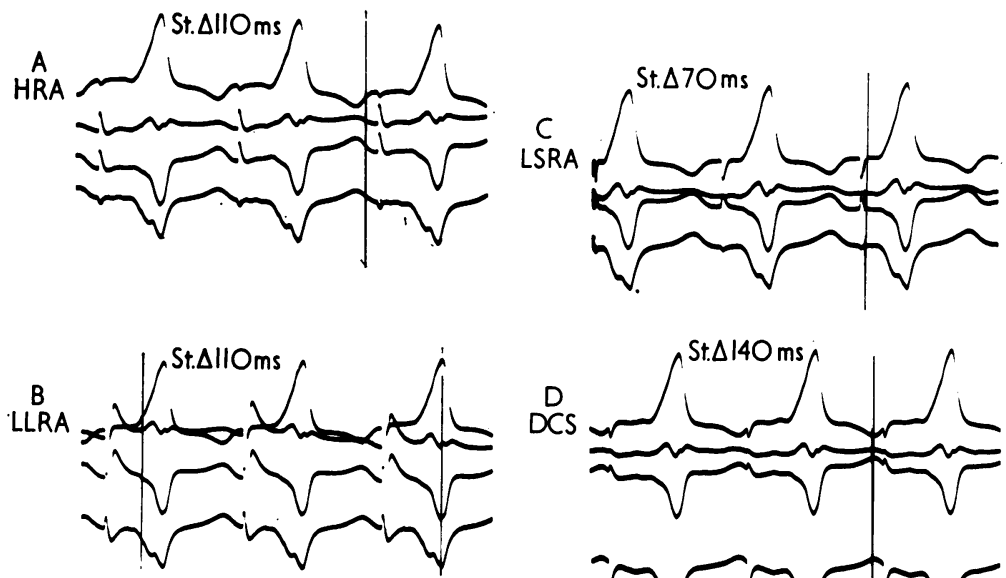

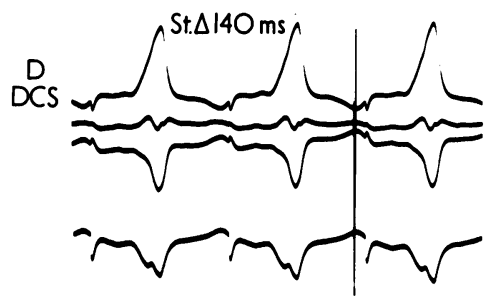

Fig. 3 The effect of atrial pacing at multiple sites on stimulus-delta intervals in case 3 with type $A B$ preexcitation. In each panel surface electrocardiographic lead $I, I I, I I I$, and VI are shown. Abbreviations are similar to Fig. 1. DCS represents distal coronary sinus pacing. Note that the shortest stimulus-delta interval ( $70 \mathrm{~ms}$ ) is obtained with low septal right atrial pacing. $\mathrm{ms}, \mathrm{PA}$ of $24 \mathrm{~ms}$, AH of $53 \mathrm{~ms}$, and an $\mathrm{HV}$ of $38 \mathrm{~ms}$.

Six atrial pacing sites were selected for pacing at a cycle length of $460 \mathrm{~ms}$. The stimulus-delta intervals and the QRS duration from these sites were as follows: (1) High right atrium, with a stimulusdelta of $130 \mathrm{~ms}$ and QRS duration of $120 \mathrm{~ms}$ (Fig. 4A). (2) Low lateral right atrium, with a stimulus-delta of $140 \mathrm{~ms}$ and QRS duration of $120 \mathrm{~ms}$. (3) Low septal right atrium, with a stimulus-delta of $100 \mathrm{~ms}$ and QRS duration of $100 \mathrm{~ms}$ (Fig. 4C). (4) Proximal coronary sinus, with a stimulus-delta of $60 \mathrm{~ms}$ and QRS duration of $140 \mathrm{~ms}$ (Fig. 4D). (5) Midcoronary sinus, with a stimulusdelta of $80 \mathrm{~ms}$ and QRS duration of $140 \mathrm{~ms}$ (Fig. 4E). (6) Distal coronary sinus with a stimulus-delta of $100 \mathrm{~ms}$ and QRS duration of $130 \mathrm{~ms}$ (Fig. 4F).

Retrograde atrial activation sequences were determined during ventricular pacing. The proximal coronary sinus atrial electrogram preceded the low septal right atrial, high right atrial, low lateral right atrial, and distal coronary sinus activation by $5,20,60$, and $30 \mathrm{~ms}$, respectively. The retrograde
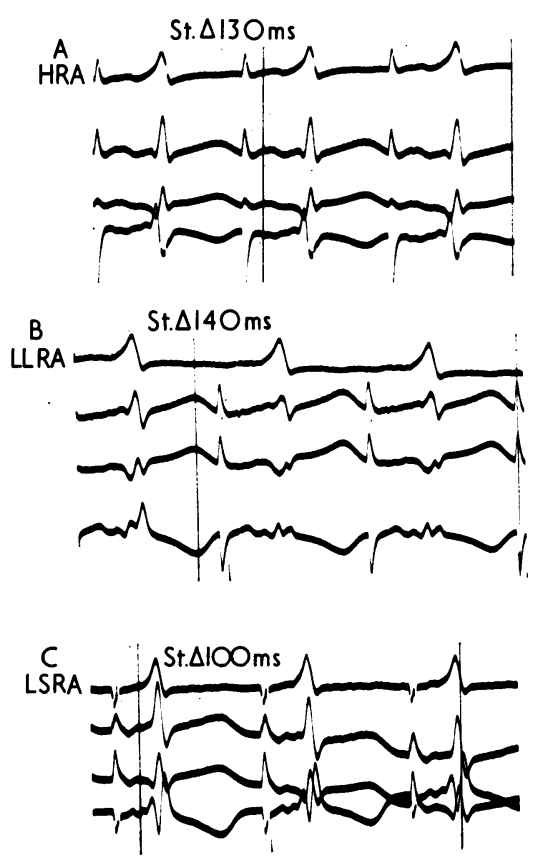
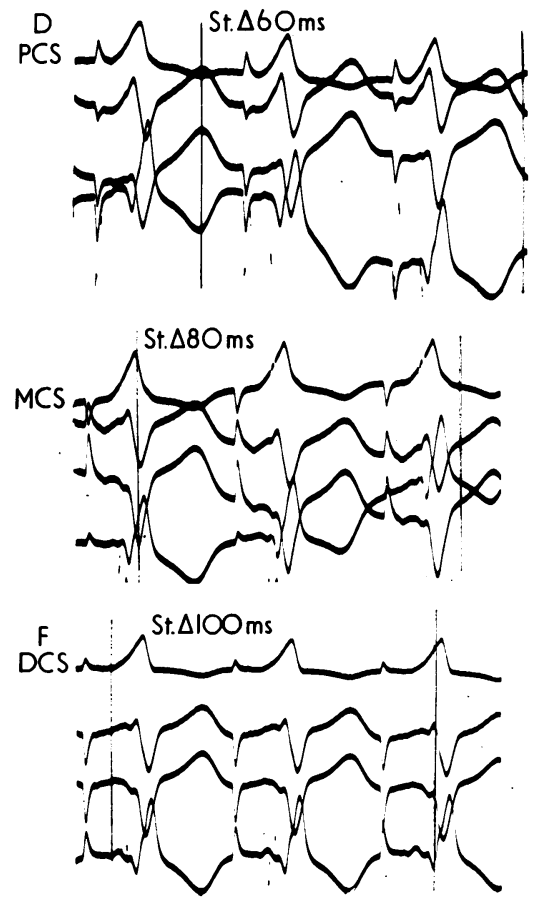

Fig. 4 The effect of atrial pacing at multiple sites on stimulus-delta intervals in case 4 with type A preexcitation. In each panel, surface electrocardiographic leads $I, I I, I I I$, and VI are shown. Abbreviations are similar to Fig. 1 and 3. PCS and MCS represent proximal and mid coronary sinus pacing. Note that the shortest stimulus-delta interval $(60 \mathrm{~ms})$ is obtained with proximal coronary sinus pacing. 
conduction time to the proximal coronary sinus site was $60 \mathrm{~ms}$ with ventricular pacing at all tested cycle lengths.

\section{Comment}

In this patient, the electrocardiogram suggested a left posterior connection. The atrial pacing site study revealed the shortest stimulus-delta and widest QRS complex from the proximal coronary sinus, suggesting a posterior left atrial connection. Though this anomalous pathway location was not confirmed with epicardial mapping and surgery, retrograde activation data provided strong confirmatory evidence for the predicted site of preexcitation (Gallagher et al., 1975).

\section{CASE 5}

This was a 53-year-old man with primary myocardial disease, subacute pericarditis, and recurrent paroxysmal atrial flutter with 1:1 anomalous pathway conduction. Electrocardiogram revealed type $\mathrm{C}$ pre-excitation (Fig. 5). Basic conduction intervals were recorded during sinus rhythm at a cycle length of $980 \mathrm{~ms}$ and revealed a $P$ delta interval of $130 \mathrm{~ms}$, and a PA of $25 \mathrm{~ms}$. AH and $\mathrm{HV}$ intervals could not be measured since the $H$ potential was buried within the ventricular electrogram.

Five stimulation sites were selected for pacing at a cycle length of $460 \mathrm{~ms}$. The stimulus-delta intervals and QRS duration from these sites were as follows: (1) High right atrium, with a stimulusdelta of $170 \mathrm{~ms}$ and QRS duration of $180 \mathrm{~ms}$ (Fig. 5A). (2) Low lateral right atrium, with a stimulusdelta of $180 \mathrm{~ms}$ and QRS duration of $180 \mathrm{~ms}$ (Fig. 5B). (3) Low septal right atrium, with a stimulusdelta of $140 \mathrm{~ms}$ and a QRS duration of $140 \mathrm{~ms}$ (Fig. 5C). (4) Proximal coronary sinus, with a stimulus-delta of $80 \mathrm{~ms}$ and QRS duration of $190 \mathrm{~ms}$ (Fig. 5D). (5) Distal coronary sinus, with a stimulus-delta of $70 \mathrm{~ms}$ and QRS duration of 190 ms (Fig. 5E).

Because of pericarditis and medically intractable recurrent atrial flutter with hypotension necessitating frequent cardioversions, surgical ablation of the anomalous connection was attempted. After pericardectomy, the ventricular epicardium was mapped. The earliest area of ventricular activation was on the posterolateral left ventricle adjacent to the AV ring, preceding the onset of the delta wave by $25 \mathrm{~ms}$ (Fig. 6). After epicardial mapping, the left atrium was entered during cardiopulmonary bypass. A transmural atrial incision was made parallel to the AV ring and adjacent to the earliest site of ventricular activation, which resulted in normalisation of the electrocardiogram with loss of the delta wave. During surgical repair, the left circumflex artery was damaged with development of intraoperative myocardial infarction. The patient expired with postoperative cardiogenic shock.
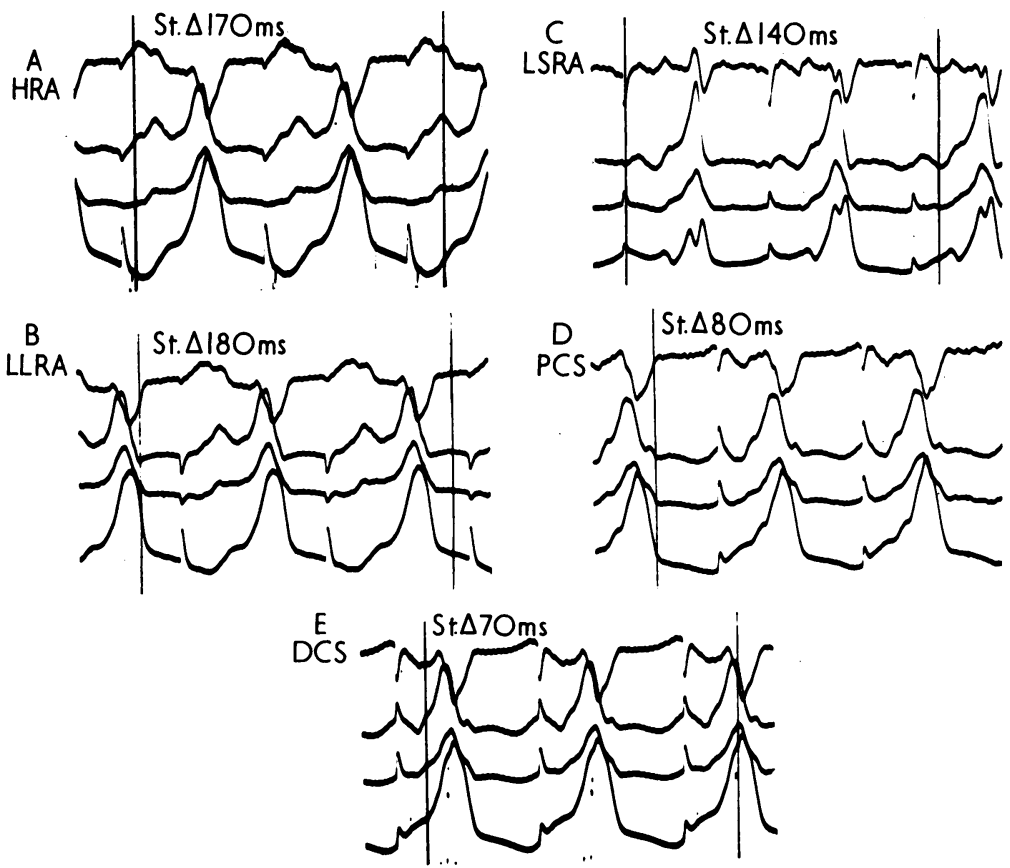

Fig. 5 The effect of atrial pacing site on stimulus-delta intervals in case 5 with type $C$ pre-excitation. In each panel, surface electrocardiographic leads $I, I I, I I I$, and V1 are shown. Abbreviations are similar to Fig. 1 and 4. Note that the shortest stimulus-delta interval ( $70 \mathrm{~ms}$ ) is obtained with distal coronary sinus pacing. 


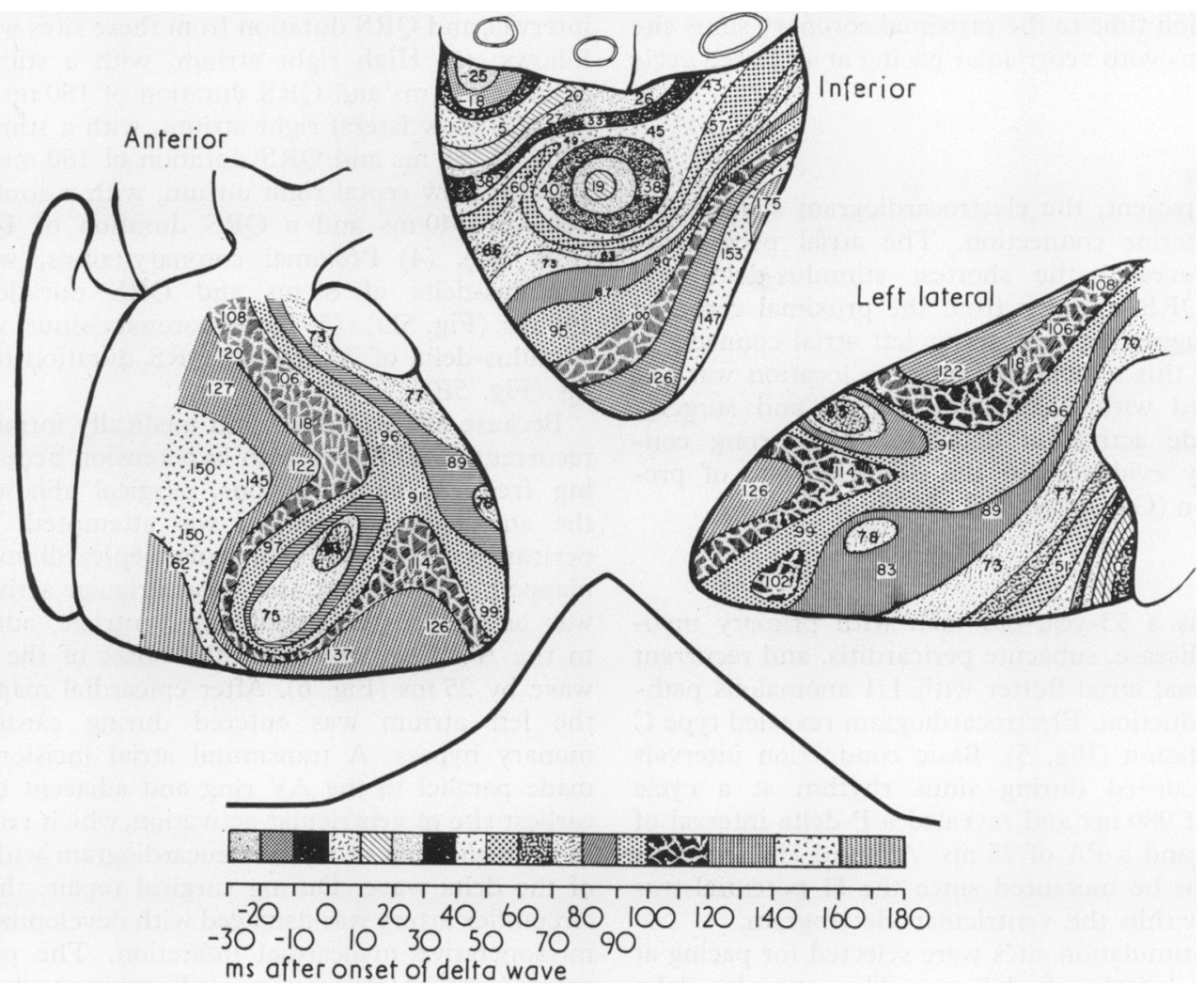

Fig. 6 Diagram of epicardial activation in case 1 during pre-excitation. Isochronous lines are drawn at 10 to $20 \mathrm{~ms}$ intervals with reference to the earliest onset of delta wave on multiple surface electrocardiographic leads. Anterior (upper), left lateral (lower left), and inferior (lower right) views of the heart are shown. The earliest area of epicardial activity is on the anterior right ventricle preceding the onset of the delta wave by $38 \mathrm{~ms}$.

\section{Comment}

The electrocardiogram during total pre-excitation suggested a left lateral anomalous connection. Multiple pacing site study strongly suggested a left lateral bypass, because of the shortest stimulusdelta $(70 \mathrm{~ms})$ with distal coronary sinus pacing. Epicardial mapping and surgery confirmed the presence of a left lateral anomalous connection.

\section{Discussion}

The success of surgery in the Wolff-ParkinsonWhite syndrome is partially determined by anomalous pathway location. In a recent report by Tonkin et al. (1975) cure of pre-excitation was achieved in 91 per cent of patients with anomalous pathways located in the free wall of the mitral or tricuspid annulus. In contrast, cure of pre-excitation was achieved in only 36 per cent of cases with septal anomalous pathways (Tonkin et al., 1975). Thus, the ability to predict the site of anomalous pathway before operation is of major importance in the presurgical evaluation of patients with preexcitation.

Electrophysiological studies in the catheterisation laboratory may allow localisation of anomalous pathway location. The means for achieving this localisation are as follows: (1) Recording of retrograde atrial activation sequences from multiple sites during AV re-entrant tachycardia or ventricular pacing, (2) observations of changes in retrograde atrial activation time during the occurrence of functional bundle-branch block, allowing lateralisation of the anomalous pathway, and (3) measurement of stimulus-delta intervals and QRS duration from multiple atrial stimulation sites.

The use of retrograde atrial sequence and of functional bundle-branch block has been described in detail in recent publications (Coumel and Attuel, 1974; Spurrell et al., 1974; Zipes et al., 1974; Svenson et al., 1974; Wellens and Durrer, 1974; Gallagher et al., 1975; Neuss et al., 1975; Tonkin 
et al., 1975). The use of atrial stimulation at multiple sites has received less attention. Wellens et al. (1971) noted that left atrial pacing produced more pre-excitation than right atrial pacing in one patient with type A pre-excitation. Touboul et al. (1972) made similar observations in 2 patients with type A Wolff - Parkinson - White syndrome. Recently, Svenson et al. (1975) reported on the electrophysiological evaluation of 25 patients with Wolff-Parkinson-White syndrome and commented that the degree of ventricular pre-excitation was greatest and the stimulus-delta interval was shortest when atrial pacing was performed from the site at which earliest atrial activation was recorded during AV re-entrant tachycardia. However, the atrial sites tested and their stimulus-delta intervals were not reported.

The present study systematically presents multiple site data in 5 patients with the Wolff-ParkinsonWhite syndrome. In each of our cases, an atrial stimulation site with shortest stimulus-delta interval could be identified, as could a site with longest stimulus-delta. Several assumptions were made in evaluating our data. First, it was assumed that anomalous pathway conduction time was constant and independent of atrial stimulation during preexcitation. Secondly, it was assumed that atrial conduction velocity was constant and independent of the site of atrial pacing. We did recognise that the anomalous pathway may respond in an all-or-none fashion depending upon the site of stimulation (Svenson et al., 1975). Accepting these assumptions, differences in stimulus-delta intervals between different stimulation sites should reflect varying distances between atrial stimulation site and the atrial insertion of the anomalous pathway. The atrial pacing site with the shortest stimulus-delta interval should correspond to a location closest to the atrial insertion of the anomalous pathway. Conversely, the longest stimulus-delta interval should be obtained from the most distant atrial pacing site.

In the present study, the shortest observed stimulus-delta interval in each of the patients was between 60 and $80 \mathrm{~ms}$. In all patients, the difference between shortest and longest stimulus-delta interval was always between 60 and $110 \mathrm{~ms}$. The hypothesis that the site with shortest stimulus delta reflected the atrial insertion of the anomalous pathway appeared to be confirmed. In 3 of our patients, one each with right anterior, right posterior, and left lateral anomalous pathway locations, this confirmation was obtained by epicardial mapping and anomalous pathway surgery. In 2 of our patients, though epicardial maps were not obtained (surgery being avoided in these patients because of the results of pacing site studies), strong supportive evidence for the predicted site of pre-excitation was obtained by use of retrograde activation data. The latter technique has been previously confirmed to be of value in predicting anomalous pathway location with epicardial mapping (Svenson et al., 1974, 1975; Gallagher et al., 1975). It should also be pointed out that multiple stimulation site studies allowed differentiation of the site of anomalous pathways in patients with similar electrocardiographic findings.

\section{CLINICAL IMPLICATIONS}

The present study suggests that atrial pacing at multiple stimulation sites provides useful information in the electrophysiological assessment of patients with Wolff-Parkinson-White syndrome who are being considered for pre-excitation surgery. The demonstration of a stimulation site with shortest stimulus-delta interval, particularly if that interval is less than $80 \mathrm{~ms}$, is strong evidence for that site being close to the atrial insertion of the anomalous pathway. The simultaneous demonstration of maximum pre-excitation at a relatively slow-paced rate from the same site provides additional supportive evidence. However, if normal pathway time is rapid from the site of the short stimulus delta, then maximum pre-excitation might not be noted (Amat-y-Leon et al., 1975).

The use of multiple pacing site data appears to be particularly useful in detecting patients with septal anomalous pathways. In this group of patients, electrocardiograms do not reveal pathognomonic findings of septal pre-excitation, but usually suggest either left or right posterior preexcitation (Tonkin et al., 1975). Studies of retrograde conduction in these patients (either with ventricular pacing, or during paroxysmal tachycardia) show early activation of the low septal right atrium (Svenson et al., 1975). Previous studies have shown that the normal sequence of retrograde atrial activation in patients without pre-excitation is characterised by earliest activation of the low right atrial septum (Amat-y-Leon et al., 1976). This is also true in patients with AV nodal re-entrant paroxysmal tachycardia, when examining the activation sequence of $\mathrm{AV}$ nodal re-entrant atrial echo beats (Amat-y-Leon et al., 1976). On theoretical grounds, one would also expect a similar retrograde activation pattern from a retrogradely conducting James tract (atrionodal connection) or atriofascicular tract (Anderson et al., 1975). Thus, demonstration of early retrograde activation of low septal right atrium would not be pathognomonic of septal pre-excitation. Demonstration of the shortest stimulus-delta interval with low right atrial septal pacing would provide strong evidence for the 
presence of an anterogradely conducting septal anomalous pathway bypassing the AV node.

The present study thus indicates that atrial pacing at multiple sites is useful in the evaluation of patients with Wolff-Parkinson-White syndrome, and supplements the other methods used for prediction of site of pre-excitation. The use of atrial sites provides relatively direct information concerning the location of anterogradely conducting anomalous pathways.

\section{References}

Amat-y-Leon, F., Denes, P., Wu, D., Pietras, R. J., and Rosen, K. M. (1975). The effects of atrial pacing site on atrial and A-V nodal function. British Heart fournal, 37, 576-582.

Amat-y-Leon, F., Dhingra, R. C., Wu, D., Denes, P., Wyndham, C., and Rosen, K. M. (1976). Catheter mapping of retrograde atrial activation. Observations during ventricular pacing and AV nodal reentrant paroxysmal tachycardia. British Heart fournal, 38, 355-362.

Anderson, R. H., Becker, A. E., Brechenmacher, C., Davies, M. J., and Rossi, L. (1975). Ventricular preexcitation: a proposed nomenclature for its substrates. European fournal of Cardiology, 3, 27-36.

Boineau, J. P., Moore, E. N., Spear, J. F., and Sealy, W. C. (1973). Basis of static and dynamic electrocardiographic variations in Wolff-Parkinson-White syndrome. American Fournal of Cardiology, 32, 32-45.

Coumel, P. H., and Attuel, P. (1974). Reciprocating tachycardia in overt and latent preexcitation. Influence of functional bundle branch block on the rate of the tachycardia. European fournal of Cardiology, 1, 423-436.

Denes, P., Wyndham, C., Dhingra, R., Wu, D., Amat-yLeon, F., Levitsky, S., and Rosen, K. M. (1974). Prediction of anomalous pathway location in patients with WolffParkinson-White syndrome using atrial pacing at multiple sites. Circulation, 49-50, Suppl. III, 222.

Denes, P., Amat-y-Leon, F., Wyndham, C., Wu, D., Levitsky, S., and Rosen, K. M. (1976a). Electrophysiologic demonstration of bilateral anomalous pathways in a patient with Wolff-Parkinson-White syndrome (type B preexcitation). American fournal of Cardiology, 37, 93-101.

Denes, P., Wyndham, C., and Rosen, K. M. (1976b). Intractable paroxysmal tachycardia caused by a concealed retrogradely conducting Kent bundle. Demonstration by epicardial mapping and cure of tachycardias by surgical interruption of the His bundle. British Heart fournal, 38, 758-763.

Gallagher, J. J., Gilbert, M., Svenson, R. H., Sealy, W. C., Kasell, J., and Wallace, A. G. (1975). Wolff-Parkinson-
White syndrome: the problem, evaluation and surgical correction. Circulation, 51, 767-785.

Neuss, H., Schlepper, M., and Thormann, J. (1975). Analysis of reentry mechanisms in those patients with concealed Wolff-Parkinson-White syndrome. Circulation, 51, 75-81.

Sealy, W. C., and Wallace, A. G. (1974). Surgical treatment of Wolff-Parkinson-White syndrome. Fournal of Thoracic and Cardiovascular Surgery, 68, 757-769.

Spurrell, R. A. J., Krikler, D. M., and Sowton, E. (1974). Retrograde invasion of the bundle branches producing aberration of the QRS complex during supraventricular tachycardia studied by programmed electrical stimulation. Circulation, 50, 487-495.

Svenson, R. H., Gallagher, J. J., Sealy, W. C., and Wallace, A. G. (1974). An electrophysiologic approach to the surgical treatment of the Wolff-Parkinson-White syndrome: a report of two cases utilizing catheter recording and epicardial mapping techniques. Circulation, 49, 799-804.

Svenson, R. H., Miller, H. C., Gallagher, J. J., and Wallace, A. G. (1975). Electrophysiological evaluation of the WolffParkinson-White syndrome. Circulation, 52, 552-562.

Tonkin, A. M., Wagner, G. S., Gallagher, J. J., Cope, G. D., Kasell, F., and Wallace, A. G. (1975). Initial forces of ventricular depolarization in the Wolff-Parkinson-White syndrome. Circulation, 52, 1030-1036.

Touboul, P., Tessier, Y., Magrina, J., Clément, C., and Delahaye, J. P. (1972). His bundle recording and electrical stimulation of atria in patients with Wolff-Parkinson-White syndrome type A. British Heart fournal, 34, 623-630.

Wellens, H. J. J., and Durrer, D. (1974). Patterns of ventriculo atrial conduction in the Wolff-Parkinson-White syndrome. Circulation, 49, 22-31.

Wellens, H. J. J., and Durrer, D. (1975). The role of an acessory atrioventricular pathway in reciprocal tachycardia. Observations in patients with and without Wolff-ParkinsonWhite syndrome. Circulation, 52, 58-72.

Wellens, H. J. J., Schuilenberg, R. M., and Durrer, D. (1971). Electrical stimulation of the heart in patients with WolffParkinson-White syndrome, type A. Circulation, 43, 99-114.

Wyndham, C., Amat-y-Leon, F., Denes, P., Dhingra, R., Burman, S. O., Pouget, J. M., and Rosen, K. M. (1974). Posterior left ventricular preexcitation. Report of a case. Archives of Internal Medicine, 134, 243-249.

Zipes, D. P., DeJoseph, R. L., and Rothbaum, D. A. (1974). Unusual properties of accessory pathways. Circulation, 49, 1200-1211.

Requests for reprints to Dr. Pablo Denes, Section of Cardiology, University of Illinois Hospital, P.O. Box 6998, Chicago, Illinois 60680, USA. 